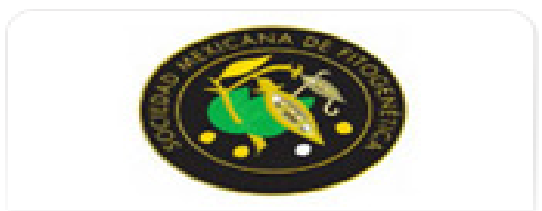

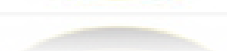

\section{Revista Fitotecnia Mexicana}

ISSN: 0187-7380

revfitotecniamex@gmail.com

Sociedad Mexicana de Fitogenética, A.C.

México

Guerrero-Aguilar, Brenda Z.; Acosta-Gallegos, Jorge A.; Sánchez-García, Bertha M.;

Ortega-Murrieta, Pedro F.; González-Chavira, Mario M.

RAZAS PATOGÉNICAS DE Fusarium oxysporum f. sp. ciceris EN GARBANZO

CULTIVADO EN GUANAJUATO, MÉXICO

Revista Fitotecnia Mexicana, vol. 38, núm. 2, 2015, pp. 183-190

Sociedad Mexicana de Fitogenética, A.C.

Chapingo, México

Disponible en: http://www.redalyc.org/articulo.oa?id=61038806008

- Cómo citar el artículo

- Número completo

- Más información del artículo

- Página de la revista en redalyc.org

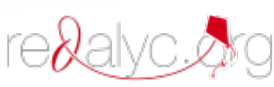

Sistema de Información Científica

Red de Revistas Científicas de América Latina, el Caribe, España y Portugal Proyecto académico sin fines de lucro, desarrollado bajo la iniciativa de acceso abierto 


\title{
RAZAS PATOGÉNICAS DE Fusarium oxysporum f. sp. ciceris EN GARBANZO CULTIVADO EN GUANAJUATO, MÉXICO
}

\author{
PATHOGENIC RACES OF Fusarium oxysporum f. sp. ciceris IN CHICKPEAS GROWN \\ IN GUANAJUATO STATE, MÉXICO
}

\author{
Brenda Z. Guerrero-Aguilar ${ }^{1}$, Jorge A. Acosta-Gallegos ${ }^{1}$, Bertha M. Sánchez-García ${ }^{1}$, \\ Pedro F. Ortega-Murrieta ${ }^{2}$ y Mario M. González-Chavira ${ }^{1 \star}$
}

\begin{abstract}
${ }^{1}$ Campo Experimental Bajío, Instituto Nacional de Investigaciones Forestales, Agrícolas y Pecuarias (INIFAP). Km. 6.5 Carr. Celaya-San Miguel Allende, A. P. 112. 38110, Celaya, Guanajuato, México. ${ }^{2}$ Campo Experimental Costa de Hermosillo. INIFAP. Blvd. del Bosque No.7 Esq. Paseo de la Pradera, Col. Valle Verde. 83200, Hermosillo, Sonora.

*Autor de correspondencia (gonzalez.mario@inifap.gob.mx)
\end{abstract}

\section{RESUMEN}

En la región de el Bajío en el centro de México, el rendimiento del garbanzo (Cicer arietinum L.) es limitado por enfermedades de la raíz como la marchitez vascular causada Fusarium oxysporum f. sp. ciceris (FOC). El conocimiento de las razas existentes de este patógeno permitirá usar los cultivares resistentes apropiados. El objetivo fue identificar las razas de FOC existentes en El Bajío, estado de Guanajuato por medio de marcadores moleculares específicos. Se analizaron aislados provenientes de cinco municipios productores de garbanzo (Celaya, Salvatierra, Valle de Santiago, Huanímaro y Pénjamo). Se probaron 12 iniciadores, siete de secuencia arbitraria tipo RAPD (OP1-01, OPI-09, OPI-18, OPF-06, OPF-10, OPF-12 y OPF-16) asociados a las razas $0,1 \mathrm{~A}, 1 \mathrm{~B} / \mathrm{C}, 2,3,4,5$ y 6 de $F$. oxysporum $\mathrm{f}$. sp. ciceris, y cinco de secuencia tipo SCAR (FocR0-M15, FocR1B/C-N5, FocR5-L10, FocR6-O2, FocR6-P18-FocR0-M15), procedentes de los fragmentos marcadores raciales tipo RAPD inicialmente identificados. Los marcadores señalaron la presencia de cuatro razas: $0,1 \mathrm{~B} / \mathrm{C}, 4$ y 6 , de FOC; las razas de mayor frecuencia fueron la 0 y $1 \mathrm{~B} / \mathrm{C}$, que inducen el síntoma de "amarillamiento", y la raza 6 que induce el síntoma de marchitez. Con las razas identificadas en Guanajuato y la raza 5 aislada en el estado de Sinaloa, se realizó una prueba de patogenicidad sobre las líneas diferenciales: JG 62, Sanford, P 2245, CRIL 1-53, CRIL 1-94, CRIL 1-17, CRIL 1-36, y WR 315, por el método de inmersión de raíces. Los resultados de la inoculación verificaron la identificación de razas con los marcadores. Las diferenciales resistentes fueron CRIL 1-36, WR 315, y CRIL 1-17, las que pueden utilizarse como progenitores en el mejoramiento de la resistencia contra FOC en México.

Palabras clave: Cicer arietinum, diversidad patogénica, marcadores moleculares, variedades diferenciales.

\section{SUMMARY}

At El Bajío region of Central México, chickpea (Cicer arietinum L.) yields are limited by root rot such as wilt caused by Fusarium oxysporum f. sp. ciceris (FOC). Knowledge on the prevailing races of this pathogen could guide efforts to use appropriate resistant cultivars. The study identified FOC races present at the El Bajío, Guanajuato State by using specific molecular markers. Isolates from five chickpea producing counties were analyzed (Celaya, Salvatierra, Valle de Santiago, Huanímaro and Pénjamo). Twelve primers were used: seven RAPD-type arbitrary sequences (OP1-01, OPI-09, OPI-18, OPF-06, OPF-10, OPF-12 and OPF-16) associated to races $0,1 \mathrm{~A}, 1 \mathrm{~B} / \mathrm{C}, 2,3,4$,
5 and 6 of F. oxysporum f. sp. ciceris; and five SCAR-type sequences (FocR0-M15, FocR1B/C-N5, FocR5-L10, FocR6-O2, and FocR6P18-FocR0-M15) derived from RAPD markers previously identified. Markers showed presence of four FOC races in Guanajuato: 0, 1B/C, 4 and 6 . Races 0 and $1 \mathrm{~B} / \mathrm{C}$ that produce the yellowing symptom, and race 6 that induces the wilting symptom, were highly frequent. A pathogenicity test was performed taking one sample from each isolated race, plus a race 5 isolate from Sinaloa State upon the differential genotypes: JG 62, Sanford, P 2245, CRIL 1-53, CRIL 1-94, CRIL 1-17, CRIL 1-36, and WR 315. Infection was promoted by immersion of the root system into liquid inoculum. The results from the inoculation test validated the racial identification made with the specific molecular markers. Since differential cultivars CRIL 1-36, WR 315 and CRIL 1-17 showed to be resistant, they could be used as parental stocks in the breeding of resistant cultivars against FOC in México.

Index words: Cicer arietinum, pathogenic diversity, molecular markers, differential cultivars.

\section{INTRODUCCIÓN}

En México la producción de garbanzo (Cicer arietinum L.) se lleva a cabo en dos regiones, el Noroeste (Sinaloa, Sonora y Baja California Sur) donde se produce garbanzo blanco o "kabuli" que se destina para exportación, con grano color blanco cremoso a blanco lechoso y calibre grande (60 a 80 g en 100 semillas), y en El Bajío (áreas de Jalisco, Michoacán y Guanajuato), en donde se produce ambos tipos, el blanco para grano o consumo en fresco y el forrajero o tipo "desi" de color café y de menor tamaño (15 a $30 \mathrm{~g}$ en 100 semillas), este último dedicado a la alimentación de ganado porcino, aves y bovino.

Las enfermedades que causan pérdidas en la producción de garbanzo son las pudriciones de raíz, y entre ellas la más importante es la fusariosis o marchitez vascular, ocasionada principalmente por Fusarium oxysporum f. sp. ciceris (FOC). La pudrición de raíz es una enfermedad de etiología compleja ya que puede haber diversos agentes causales involucrados. Según Trapero-Casas y Jiménez-Díaz (1985a), 
la fusariosis vascular causada por FOC se caracteriza por dos síntomas distinguibles "marchitez vascular" y "amarillamiento vascular". Ambos síntomas son consecuencia de infecciones vasculares en la planta que llevan asociada una coloración castaño oscuro del xilema, y ocasionalmente de la médula de la raíz, cuello y tallo de las plantas infectadas (Trapero-Casas y Jiménez-Díaz, 1985b).

El síntoma de amarillamiento vascular se expresa con más lentitud que el de marchitez vascular y se caracteriza por el desarrollo de clorosis, amarillamiento y necrosis de los foliolos de las hojas inferiores, que dan lugar a la defoliación prematura de éstas. En experimentos en ambiente controlado, las plantas susceptibles generalmente mueren después de transcurridos $40 \mathrm{~d}$ después la siembra en un suelo infestado artificialmente (Trapero-Casas y Jiménez -Díaz 1985b). El síntoma de marchitez vascular se caracteriza por el desarrollo rápido de flacidez de las hojas en cualquier nivel de la planta y la desecación de foliolos que adquieren una coloración verde-grisácea, que luego se extiende a toda la planta. La flacidez es seguida por desecación de hojas y tallos, que adquieren un color castaño claro y finalmente se produce la muerte de la planta (Trapero-Casas y Jiménez-Díaz, 1985b). En inoculaciones artificiales, la muerte de plantas susceptibles en suelo infestado ocurre generalmente antes de transcurridos $20 \mathrm{~d}$ después de la siembra (Trapero-Casas y Jiménez-Díaz, 1985a).

En un principio las razas de FOC se identificaron con un conjunto de variedades diferenciales (Haware y Nene, 1982; Sharma et al., 2005), posteriormente se desarrolló una forma eficaz para identificarlas por medio de marcadores moleculares específicos. La identificación de las razas de FOC por patogenicidad y severidad ha sido complicada y confusa, por la diversidad de variedades diferenciales utilizadas por diferentes grupos de trabajo, las cuales han variado desde 8 hasta 22 (Haware y Nene, 1982; Phillips 1988; Jiménez-Díaz et al., 1989; Tullu, 1998; Sharma et al., 2005). Otro factor que ha complicado el proceso de determinación de razas es el criterio inconsistente en definir la susceptibilidad y la resistencia. Las escalas de severidad de la enfermedad han utilizado el fenotipo de la resistencia y susceptibilidad para la identificación de las razas, en donde cada autor ha variado considerablemente esas escalas (Cuadro 1).

Por la inconsistencia de las variedades diferenciales y de las escalas de severidad usadas, en los últimos años se ha implementado una herramienta que permite la identificación de las razas de FOC mediante el uso de marcadores de ADN (Jiménez-Gasco y Jiménez Díaz, 2003; Arvayo-Ortiz et al., 2011). Los marcadores utilizados fueron desarrollados a partir de fragmentos tipo RAPD amplificados al azar. Después de su amplificación los fragmentos fueron seleccionados y secuenciados para corroborar la especificidad de las bandas de $\mathrm{ADN}$ a las razas 0, 1B/C, 2, 3, 4, 5, y 6 . Posteriormente, estas secuencias fueron convertidas a marcadores tipo SCAR (Jiménez-Gasco y Jiménez Díaz, 2003).

El objetivo de este estudio fue identificar las razas de $F$. oxysporum f. sp. ciceris que afectan el cultivo de garbanzo en el estado de Guanajuato mediante marcadores moleculares tipo RAPD y SCAR, y verificar la patogenicidad de los aislamientos en variedades diferenciales de garbanzo.

\section{MATERIALES Y MÉTODOS}

En 2011 y 2012 se hicieron muestreos en campos comerciales y experimentales en cinco municipios de El Bajío Guanajuatense ubicado en los municipios de: Salvatierra, Huanímaro, Valle de Santiago, Pénjamo y Celaya (Cuadro 2). Se muestrearon 21 lotes y se colectaron 10 plantas de garbanzo tomadas al azar con síntomas de marchitamiento y amarillamiento ocasionados por Fusarium sp. para un total de 210 muestras. Las colectas de plantas se hicieron en diferentes etapas de crecimiento, tal como fueron localizadas en los campos. Las coordenadas de los sitios de colecta se determinaron con un posicionador geográfico (Garmin Etrex 20®; Kansas, USA) y la clasificación de suelos se hizo con un mapa de suelos elaborado por el Soil Survey Staff de Estados Unidos de América (Departamento de Agricultura de los Estados Unidos, USDA).

Cuadro 1. Escalas de severidad de la enfermedad que han sido usadas para el estudio de las razas de Fusarium oxysporum f. sp. ciceris.

\begin{tabular}{lccc}
\hline \multirow{2}{*}{ Fuente } & \multicolumn{3}{c}{ Escala de severidad (\% de marchitez) } \\
\cline { 2 - 4 } & Resistente & Moderadamente susceptible & Susceptible \\
\hline Haware y Nene, 1982 & $0-20$ & $21-50$ & $>50$ \\
Phillips, 1988 & $0-20$ & $21-50$ & $>50$ \\
Jiménez-Díaz et al., 1989 & $0-33$ & $34-66$ & $67-100$ \\
Sharma et al., 2005 & $1-10$ & $11-50$ & $51-100$ \\
Tullu, 1998 & $1-10$ & $11-90$ & $91-100$ \\
\hline
\end{tabular}

Reacción de enfermedad: media; Reacción de la enfermedad: intermedia. 
Cuadro 2. Información geográfica y tipo de suelo de las localidades de Guanajuato donde se colectaron plantas de garbanzo en diferentes etapas de crecimiento y con síntomas asociados a FOC.

\begin{tabular}{|c|c|c|c|c|c|c|}
\hline Municipio & Coordenadas & Altitud (m) & $\begin{array}{c}\text { Tipo de } \\
\text { suelo }\end{array}$ & Genotipo & Etapa de crecimiento & Síntomas observados \\
\hline Huanímaro & $\begin{array}{c}20^{\circ} 21^{\prime} 17.1^{\prime \prime} \mathrm{N} \\
101^{\circ} 25^{\prime} 31.3^{\prime \prime} \mathrm{O}\end{array}$ & 1690 & Litosol & Bco. Sinaloa & Llenado de vaina & $\begin{array}{c}\text { Marchitez y } \\
\text { amarillamiento }\end{array}$ \\
\hline $\begin{array}{l}\text { Valle de } \\
\text { Santiago }\end{array}$ & $\begin{array}{c}20^{\circ} 25^{\prime} 02.4^{\prime \prime} \mathrm{N} \\
101^{\circ} 6^{\prime} 4.0^{\prime \prime} \mathrm{O}\end{array}$ & 1715 & $\begin{array}{l}\text { Vertisol } \\
\text { pélico }\end{array}$ & Bco. Sinaloa & Llenado de vaina & $\begin{array}{c}\text { Marchitez y } \\
\text { amarillamiento }\end{array}$ \\
\hline Salvatierra & $\begin{array}{c}20^{\circ} 13^{\prime} 05.4^{\prime \prime} \mathrm{N} \\
100^{\circ} 53^{\prime} 44.3^{\prime \prime} \mathrm{O}\end{array}$ & 1691 & $\begin{array}{l}\text { Vertisol } \\
\text { pélico }\end{array}$ & Bco. Sinaloa & Madurez & $\begin{array}{c}\text { Marchitez y } \\
\text { amarillamiento }\end{array}$ \\
\hline Pénjamo & $\begin{array}{l}20^{\circ} 17^{\prime} 99.6^{\prime \prime} \mathrm{N} \\
10^{\circ} 48^{\prime} 32.7^{\prime \prime} \mathrm{O}\end{array}$ & 1705 & $\begin{array}{l}\text { Vertisol } \\
\text { pélico }\end{array}$ & Bco. Sinaloa & Madurez & Marchitez \\
\hline Celaya & $\begin{array}{c}20^{\circ} 35^{\prime} 20.12^{\prime \prime} \mathrm{N} \\
100^{\circ} 49^{\prime} 41.31^{\prime \prime O}\end{array}$ & 1760 & Vertisol & $\begin{array}{l}\text { Varios } \\
\text { genotipos }\end{array}$ & Floración & $\begin{array}{c}\text { Marchitez y } \\
\text { amarillamiento }\end{array}$ \\
\hline
\end{tabular}

\section{Aislamiento, purificación e identificación de especies de Fusarium oxysporum f. sp. ciceris}

En las plantas colectadas se tomaron muestras de tejido mediante cortes longitudinales de $3 \mathrm{~mm}$ de raíz y tallo en donde se observó la lesión necrótica. El tejido obtenido se desinfectó con hipoclorito de sodio $5 \%$ por 1 min y se enjuagó con agua destilada estéril. Se tomaron 5 fragmentos de tejido de raíz y tallo y se colocaron por separado en cajas Petri (90 x 15) con medio agar papa dextrosa (APD) acidificado $\left(200 \mu \mathrm{L} \mathrm{L}^{-1}\right.$ de ácido láctico a $\left.85 \%\right)$, y se incubaron a temperatura ambiente $\left(22\right.$ a $\left.25^{\circ} \mathrm{C}\right)$. Los aislados obtenidos se purificaron mediante cultivos monospóricos; para ello se agregó un fragmento de $2 \mathrm{~cm}$ de hifas en un microtubo de $1.5 \mathrm{~mL}$ con $1 \mathrm{~mL}$ de agua destilada estéril, se tomaron $30 \mu \mathrm{L}$ de la suspensión y con ayuda de una varilla de vidrio se esparció en cajas con medio agua-agar. Se incubaron por un periodo de $24 \mathrm{~h}$ a temperatura ambiente $\left(22\right.$ a $\left.25^{\circ} \mathrm{C}\right)$. Con un microscopio Leica modelo DME ${ }^{\circledR}$ (Alemania) se observó la germinación de los conidios y se transfirieron tres de ellos a medio APD, donde los cultivos monoconidiales fueron mantenidos en activo y utilizados para llevar acabo la identificación de especie.

Para la identificación de Fusarium a nivel especie los cultivos monoconidiales se sembraron en tres medios de cultivo: Agar Papa Dextrosa (APD), Spezieller Nahrstoffmmarmer (SNA; $1.0 \mathrm{~g}$ de $\mathrm{KH}_{2} \mathrm{PO}_{4}$. $1.0 \mathrm{~g}$ de $\mathrm{KNO}_{3}, 0.5 \mathrm{~g}$ de $\mathrm{MgSO}_{4} .7 \mathrm{H}_{2} \mathrm{O}, 0.5 \mathrm{~g}$ de $\mathrm{KCl}, 0.2 \mathrm{~g}$ de glucosa, $0.2 \mathrm{~g}$ de sacarosa y $20 \mathrm{~g}$ de agar en $1 \mathrm{~L}$ de agua destilada) (Nirenberg, 1976) y en hojas de clavel (Dianthus caryophyllus L.) o plátano (Musa sp.)(CLA; $20 \mathrm{~g}$ de agar en $1 \mathrm{~L}$ de agua destilada luego se colocaron de cuatro a diez piezas de hojas de clavel estériles en la superficie del medio agua-agar justo antes de que el medio solidificara). Una vez preparados los medios se colocó un fragmento de micelio en cada uno y se incubó a temperatura ambiente. Las colonias crecidas se observaron al microscopio Leica DME® (Alemania) (4X, 10X, 40X), la identificación se basó en las claves de Leslie y Summerell (2006), con base en características como: presencia de color, tasa de crecimiento, micelio aéreo, pigmentación del medio y formación de estructuras fúngicas (macroconidios, microconidios y clamidosporas). Los aislados identificados se conservaron en medio SNA con aceite mineral, a $4.0^{\circ} \mathrm{C}$ (Massa 1991).

\section{Extracción de ADN de Fusarium f. sp. ciceris}

Para la extracción de ADN fúngico se utilizó el método descrito por Raeder y Broda (1985), con ligeras modificaciones. Se colocó un fragmento de micelio de $0.3 \mathrm{~cm}$ de los cultivos monospóricos activos sobre una película estéril de celofán extendida sobre una placa del medio APD (Difco (®). Se incubó de 5 a $7 \mathrm{~d}$ a temperatura ambiente. Después se raspó con un bisturí estéril y se procedió a la extracción de ADN.

La identificación de razas se hizo mediante la amplificación de ADN genómico de cada uno de los aislados, con marcadores específicos para cada raza (Cuadro 3). Para ello se estandarizaron las condiciones de la reacción de PCR, mediante la determinación de las temperaturas óptimas para la amplificación del ADN con cada marcador, las cuales se presentan en una columna de cada cuadro.

La mezcla de reacción fue de $25 \mu \mathrm{L}$, preparada con $5 \mu \mathrm{M}$ de iniciador, $2.5 \mu \mathrm{M}$ dNTP, 10X amortiguador de reacción (50 $\mu \mathrm{M} \mathrm{KCl}, 10 \mathrm{mM}$ Tris-HCl, pH $\left.9.0\left[25^{\circ} \mathrm{C}\right]\right) 1 \mathrm{U}$ de Taq ADN polimerasa; $1.5 \mathrm{mM}$ de $\mathrm{MgCl}$, y $45 \mathrm{ng}$ de $\mathrm{ADN}$ genómico fúngico. Los ciclos para el PCR de los iniciadores OPF y OPI 
Cuadro 3. Iniciadores tipo SCARs y RAPDs, tamaño de fragmento esperado y temperatura de hibridación utilizados para la identificación de razas de Fusarium oxysporum f. sp. ciceris en garbanzo.

\begin{tabular}{|c|c|c|c|c|}
\hline Iniciador $^{\dagger}$ & Secuencia $\left(5^{\prime}-3^{\prime}\right)$ & Raza & Amplicon $(\mathrm{Kb})$ & $\operatorname{Tm}\left({ }^{\circ} \mathrm{C}\right)$ \\
\hline Foc0-12fwd ${ }^{\dagger \dagger}$ & GGCGTTTCGCAGCCTTACAATGAAG & \multirow{2}{*}{ F.o. ciceris } & \multirow{2}{*}{1.5} & \multirow{2}{*}{58} \\
\hline Foc0-12rev & GACTCCTTTTTCCCGAGGTAGGTCAGAT & & & \\
\hline FocR0M15fwd ${ }^{\dagger \dagger}$ & GGAGAGCAGGACAGCAAAGACTA & \multirow{2}{*}{0} & \multirow{2}{*}{9.0} & \multirow{2}{*}{61} \\
\hline FocR0M15rev & GGAGAGCAGCTACCCTAGATACACC & & & \\
\hline FocR1B/CN5fwd $\mathrm{d}^{\dagger \dagger}$ & GAGAGCAGGGTCAGCGTAGATAG & \multirow{2}{*}{$1 \mathrm{~B} / \mathrm{C}$} & \multirow{2}{*}{5,0} & \multirow{2}{*}{61} \\
\hline FocR1B/CN5rev & GCAGCAGAAGAGGAAGAAAATGTA & & & \\
\hline FocR5-L10fwd ${ }^{\Phi}$ & GGAAGCTTGGCATGACATAC & \multirow{2}{*}{5} & \multirow{2}{*}{9.0} & PCR touchdown \\
\hline FocR5-L10rev & AAGCTTGGGCACCCTCTT & & & $71-61$ \\
\hline FocR6-O2fwd ${ }^{\dagger \dagger}$ & GAGCAGTCAATGGCAATGG & \multirow{2}{*}{6} & \multirow{2}{*}{1.0} & \multirow{2}{*}{61} \\
\hline FocR6-O2rev & AGAGCAGGGTCAGCGTAGATA & & & \\
\hline FocR6-P18fwd ${ }^{\Phi}$ & GGAGAGCAGTAGAGTTACAGCAGTATT & \multirow{2}{*}{$1 \mathrm{~A}, 6$} & \multirow{2}{*}{1.5} & \multirow{2}{*}{61} \\
\hline FocR0-M15rev & GGAGAGCAGCTACCCTAGATACACC & & & \\
\hline \multirow{3}{*}{ OPI- $01^{\dagger \dagger}$} & \multirow{3}{*}{ ACCTGGACAC } & $1 \mathrm{~A}$ & 1.0 & \multirow{3}{*}{40} \\
\hline & & $0,1 \mathrm{~B} / \mathrm{C}, 6$ & 1.2 & \\
\hline & & $1 \mathrm{~A}, 6$ & 2.7 & \\
\hline \multirow{3}{*}{ OPI- $09^{\dagger \dagger}$} & \multirow{3}{*}{ TGGAGAGCAG } & $1 \mathrm{~B} / \mathrm{C}$ & 5.3 & \multirow{3}{*}{40} \\
\hline & & 0 & 9.0 & \\
\hline & & 6 & 1.3 & \\
\hline \multirow{2}{*}{ OPI-18 $8^{\Phi}$} & \multirow{2}{*}{ TGCCCAGCCT } & $1 \mathrm{~B} / \mathrm{C}$ & 5.3 & \multirow{2}{*}{40} \\
\hline & & $2,3,4$ & 1.0 & \\
\hline \multirow{2}{*}{ OPF- $06^{\Phi}$} & \multirow{2}{*}{ GGGAATTCGG } & $1 \mathrm{~B} / \mathrm{C}$ & 1.9 & \multirow{2}{*}{40} \\
\hline & & 3 & 2.0 & \\
\hline \multirow{4}{*}{ OPF- $10^{\dagger \dagger}$} & \multirow{4}{*}{ GGAAGCTTGG } & $1 \mathrm{~B} / \mathrm{C}$ & 5.1 & \multirow{4}{*}{40} \\
\hline & & 4 & 9.5 & \\
\hline & & 5 & 9.0 & \\
\hline & & $1 \mathrm{~B} / \mathrm{C}$ & 1.10 & \\
\hline OPF- $12^{\dagger \dagger}$ & ACGGTACCAG & 0 & 3.9 & 40 \\
\hline & & 2 & 9.0 & \\
\hline OPF-16 ${ }^{\Phi}$ & GGAGTACTGG & 0 & 6.5 & 40 \\
\hline
\end{tabular}

${ }^{\dagger}$ Los iniciadores fueron diseñados por Jiménez-Gasco y Jiménez-Díaz (2003). ${ }^{\dagger}$ Iniciadores o combinación de iniciadores que amplificaron. ${ }^{\Phi}$ Iniciadores o combinación de iniciadores que no amplificaron. PCR-touchdown = amplificación con rampas decrecientes de temperaturas; fwd = sentido de 5'-3'; rev = antisentido de 3 '- 5 '.

fueron: 4 min a $94{ }^{\circ} \mathrm{C}, 30$ ciclos de $1 \min$ a $94{ }^{\circ} \mathrm{C}, 1 \mathrm{~min}$ a $40{ }^{\circ} \mathrm{C}$, y 3 min a $72{ }^{\circ} \mathrm{C}$; un ciclo final de 7 min a $72{ }^{\circ} \mathrm{C}$. Para los iniciadores Foc: 2 min a $94^{\circ} \mathrm{C}, 25$ ó 28 ciclos de $30 \mathrm{~s}$ a $94^{\circ} \mathrm{C}, 1 \mathrm{~min}$ a $\mathrm{Tm}, 30 \mathrm{~s}$ a $72^{\circ} \mathrm{C}$, un ciclo final de 4 min a 72 ${ }^{\circ} \mathrm{C}$. El ciclo de PCR touch-down (Don et al., 1991) estuvo compuesto por: $2 \min$ de $94^{\circ} \mathrm{C}, 10$ ciclos a [30 s a $94^{\circ} \mathrm{C}, 1$ $\min$ a $71^{\circ} \mathrm{C}-61^{\circ} \mathrm{C}, 30 \mathrm{~s}$ a $72^{\circ} \mathrm{C}$ ] +15 ciclos [30 s a $94^{\circ} \mathrm{C}, 1$ min a $61^{\circ} \mathrm{C}, 30 \mathrm{~s}$ a $72{ }^{\circ} \mathrm{C}$ ] y 4 min de extensión final a $72{ }^{\circ} \mathrm{C}$.

\section{Pruebas de patogenicidad}

Material biológico. Se seleccionaron al azar tres aislados de diferentes razas de Fusarium oxysporum f. sp. cice- ris, identificadas por medio de marcadores moleculares, provenientes de las localidades de Valle de Santiago Foc12 (Raza 6) que ocasiona síntomas de marchitez, de Salvatierra Foc27 (Raza 1B/C) y de Pénjamo Foc71 (Raza 0) que ocasionan síntomas de amarillamiento. Se inocularon en ocho variedades diferenciales de garbanzo utilizadas por Sharma y Muehbauer (2007), JG 62, Stanford, P 2245, CRIL 1-53, CRIL 1-94, CRIL 1-17, CRIL 1-36 y WR 315.

Preparación del inoculo. La producción de inoculo se hizo a partir de las colonias almacenadas en medio SNA. Se transfirió un fragmento de cada colonia a cajas Petri con medio agar papa dextrosa acidificado $\left(200 \mu \mathrm{L} \mathrm{L}^{-1}\right.$ de ácido 
láctico a $85 \%$ ) y se mantuvieron a temperatura ambiente $\left(25^{\circ} \mathrm{C}\right)$ por un periodo de $10 \mathrm{~d}$. Se obtuvo la suspensión de conidios de cada aislado y con la ayuda de un hematocímetro Neubauer ${ }^{\circledR}$ improved, Marienfeld (Alemania) se registró una concentración de $1 \times 10^{6}$ conidios $\mathrm{mL}^{-1}$. Esta concentración de inoculación ha sido utilizada previamente por Tekeoglu et al. (2000) y Sharma et al. (2004) en garbanzo.

Inoculación. En condiciones de invernadero se sembraron siete semillas de cada una de las diferenciales en macetas de plástico $(61 \times 19 \times 17 \mathrm{~cm})$ llenadas con sustrato "peat moss" Sunshine mezcla 3. Las plantas desarrolladas se inocularon en la fase vegetativa V3 (23 a 30 d). Para la prueba de inoculación se utilizó el método de inmersión de raíces (Trapero-Casas y Jiménez Díaz, 1985a; Sharma et al., 2005) que consiste en sacar cuidadosamente las plantas evitando dañar las raíces. Se cortaron $2 \mathrm{~cm}$ aproximadamente de las puntas de las raíces y se sumergieron en la suspensión de 1 $\mathrm{x} 10^{6}$ conidios $\mathrm{mL}^{-1}$ durante $10 \mathrm{~min}$. Las plantas inoculadas se sembraron nuevamente en el macetero original.

A los 20 d después de la inoculación (DDI) las plantas se evaluaron con la escala visual de daño descrita por Schoonhoven y Pastor-Corrales (1987). Se utilizó esta escala por su simplicidad y sencillez, además de tener un porcentaje específico de síntomas o de daño que corresponde a un número fijo en la escala, por lo que a cada número corresponde una descripción exclusiva y precisa. La escala utiliza un rango de calificación de $1=$ sin síntomas aparentes de la enfermedad, hasta 9 = síntomas severos o muerte. En este trabajo se consideraron los valores de 1 a 3 como reacción resistente, los de 4 a 6 como reacción intermedia y los de 7 a 9 como reacción susceptible (Cuadro 4).

\section{RESULTADOS Y DISCUSIÓN}

\section{Razas de F. oxysporum identificadas}

De los 12 iniciadores probados para la identificación de razas, solamente siete amplificaron los fragmentos reportados por Jiménez-Gasco y Jiménez Díaz (2003); los cinco restantes no amplificaron ningún fragmento a pesar de utilizar las mismas condiciones reportadas. Esto pudo ser debido a la variación en los reactivos y el tipo de termociclador utilizados para la reacción de PCR. Por ello se utilizaron diferentes iniciadores que identificaban las mismas razas lo cual permitió su caracterización (Cuadro 3).

Se consideró que fragmentos de ADN genómico amplificados con la misma movilidad (peso molecular) correspondían al fragmento esperado, de acuerdo con Jiménez-Gasco y Jiménez Díaz (2003). Los amplicones obtenidos correspondieron sin ambigüedades a los tamaños esperados para las razas de FOC reportados por Jiménez-Gasco y Jiménez Díaz (2003). Así, el análisis con marcadores moleculares específicos identificó 33 aislados con el fragmento de $900 \mathrm{pb}$ correspondiente a la raza 0 , y 38 con el fragmento de 500 $\mathrm{pb}$ de la raza $1 \mathrm{~B} / \mathrm{C}$, razas que inducen el síntoma de amarillamiento (Trapero-Casas y Jiménez-Díaz, 1985b). Además, los resultados señalaron a 22 aislados con el fragmento de $1000 \mathrm{pb}$ de la raza 6, y seis aislados con un fragmento de 950 $\mathrm{pb}$ correspondiente a la de la raza 4 , las cuales son causantes del síntoma de marchitez.

Las razas 0 y $1 \mathrm{~B} / \mathrm{C}$ se encontraron en los cinco municipios muestreados en el estado de Guanajuato: Pénjamo, Huanímaro, Salvatierra, Valle de Santiago y Celaya; esto sugiere que son las razas de mayor distribución en el estado

Cuadro 4. Escala utilizada para evaluar la respuesta de pudrición de raíz ocasionado por Fusarium sp. (Schoonhoven y Pastor-Corrales, 1987).

\begin{tabular}{ll}
\hline Escala & Nivel de resistencia \\
\hline 1 & Ningún síntoma visible de la enfermedad $0 \%$ \\
2 & 1 a $5 \%$ de los síntomas de la enfermedad. \\
3 & $\begin{array}{l}\text { Muy pocas hojas marchitas (1 a } 3 \text { hojas, que representan no más de } 10 \% \text { del follaje), combinado con decoloración } \\
\text { vascular limitada a la raíz e hipocótilo (6 a } 10 \%) .\end{array}$ \\
4 & $\begin{array}{l}11 \text { a } 25 \% \\
5\end{array}$ \\
6 & $\begin{array}{l}\text { Aproximadamente } 25 \% \text { de hojas y ramas exhiben marchitez, y clorosis de } 26 \text { a } 35 \% \\
36 \text { a } 50 \%\end{array}$ \\
7 & $\begin{array}{l}\text { Aproximadamente } 50 \% \text { de hojas ramas exhiben marchitez, clorosis y necrosis limitada. Las plantas son achapa- } \\
\text { rradas. } 51 \text { a } 65 \%\end{array}$ \\
& 66 a $75 \%$ \\
9 & $\begin{array}{l}\text { Aproximadamente } 75 \% \text { o más de la hoja y ramas exhiben marchitez, achaparramiento severo y necrosis, con } \\
\text { defoliación prematura que frecuentemente resulta en muerte de la planta. }\end{array}$ \\
\hline
\end{tabular}


y probablemente en estados vecinos que también producen garbanzo en localidades con clima similar, como el norte de Michoacán y centro y noreste de Jalisco (Soltero, et al., 2008). La raza 0 mostró alta frecuencia en campos cultivados con garbanzo en Sinaloa y Sonora, 41 \% en 91 aislados (Arvayo-Ortiz et al., 2012). La alta presencia de esta raza en Guanajuato podría tener relación con la gran cantidad de semilla o grano que se utiliza como semilla de la variedad Blanco Sinaloa 92, susceptible a esa raza (Arvayo-Ortiz et al., 2012), semilla que se introduce al estado de Guanajuato desde la región Noroeste: Sinaloa, Sonora y Baja California Sur.

La raza 6 se ubicó en cuatro municipalidades: Salvatierra, Valle de Santiago, Pénjamo y Celaya. La raza 4 que es la primera vez que se reporta en Guanajuato estuvo presente en tres municipios: Salvatierra, Pénjamo y Celaya. La raza 5 reportada como la más agresiva a nivel mundial (Jiménez-Gasco et al., 2001) y que es la que ha ocasionado más pérdidas en este cultivo en los estados de Sonora y Sinaloa (Arvayo-Ortiz et al., 2012), no se identificó en el estado de Guanajuato, mientras que las razas 1B/C y 6 se encuentran en ambas regiones, El Bajío y el Noroeste (Arvayo-Ortiz et al., 2012). Las razas 1A, 2 y 3 no están presentes en el estado (Cuadro 5).

Los resultados anteriores indican que los marcadores de ADN específicos son una buena herramienta de apoyo para la detección temprana de la posible introducción de nuevas razas, así como para la identificación de los cambios que puedan ocurrir en las poblaciones existentes de FOC en respuesta al uso de cultivares de garbanzo resistentes a las razas prevalecientes en el estado; esto es acorde con la abundante diversidad molecular observada entre las poblaciones de FOC (Sivaramakrishnan et al., 2002).

\section{Inoculación de las variedades diferenciales}

La reacción de las diferenciales JG 62, P 2245 y W 315, coinciden con lo reportado para los aislados de la raza 0 , 1B/C y 6 (Jiménez-Fernández, 2011). Estos resultados de patogenicidad confirman los resultados moleculares, similares a los obtenidos por Jiménez-Fernández et al. (2011).

En cuanto a las fuentes de resistencia, las variedades diferenciales CRIL 1-17 y WR 315 resultaron resistentes a todas las razas inoculadas, que incluyen a la raza 5 (dato no mostrado), resultados similares a los de Sharma et al. (2005). En cambio JG 62 fue altamente susceptible a todas las razas de F.oxysporum f. sp. ciceris excepto a la raza 0; P 2245 y CRIL 1-94 fueron altamente susceptibles a todas las razas en estudio, Sanford fue susceptible a la raza 5 e intermedia a las otras tres razas; la diferencial CRIL 1-53 fue resistente a la raza 0 e intermedia a las otras tres razas.

Para el mejoramiento genético de la resistencia a FOC, las diferenciales WR 315, CRIL 1-17 y CRIL 1-36 pueden utilizarse para derivar progenies resistentes para ambas regiones, El Bajío Guanajuatense y las áreas productoras de Sinaloa. WR 315 es de grano café, tipo forrajero (desi), mientras que las líneas CRIL son de tamaño de grano medio color cremoso (kabuli). Es probable que el uso de estas últimas permita la recuperación del tipo de grano blanco cremoso con mayor facilidad que al utilizar WR 315; en ambos casos, el uso de retrocruzas sería recomendado (Fehr, 1987) para la recuperación de la mayoría de las características ventajosas de variedades y materiales avanzados en el programa de mejoramiento del Instituto Nacional de Investigaciones Forestales, Agrícolas y Pecuarias (INIFAP)(Cuadro 6).

\section{CONCLUSIONES}

Por medio de marcadores moleculares de ADN se identificaron cuatro razas de FOC que atacan al garbanzo en el estado de Guanajuato $(0,1 \mathrm{~B} / \mathrm{C}, 6$ y 4). La reacción de patogenicidad con las variedades diferenciales confirmaron los resultados obtenidos con los marcadores moleculares.

Cuadro 5. Aislados por raza de Fusarium oxysporum f. sp. ciceris en cinco municipios productores de garbanzo de Guanajuato.

\begin{tabular}{lrrrccccc}
\hline \multirow{2}{*}{ Municipio/Razas } & 0 & $1 \mathrm{~A}$ & $1 \mathrm{~B} / \mathrm{C}$ & 2 & 3 & 4 & 5 & 6 \\
\cline { 2 - 8 } & & \multicolumn{7}{c}{ Número de aislados por raza } \\
\hline Salvatierra & 6 & 0 & 15 & 0 & 0 & 2 & 0 & 14 \\
Celaya & 13 & 0 & 14 & 0 & 0 & 2 & 0 & 3 \\
Valle de Santiago & 7 & 0 & 5 & 0 & 0 & 0 & 0 & 3 \\
Huanímaro & 3 & 0 & 2 & 0 & 0 & 0 & 0 & 0 \\
Pénjamo & 4 & 0 & 2 & 0 & 0 & 2 & 0 & 2 \\
Total & 33 & 0 & 38 & 0 & 0 & 6 & 0 & 22 \\
\hline
\end{tabular}


Cuadro 6. Evaluación de ocho líneas de diferenciales de garbanzo (Cicer arietinum) y su reacción a cuatro razas de Fusarium oxysporum f. sp. ciceris.

\begin{tabular}{|c|c|c|c|c|c|c|c|}
\hline \multirow{3}{*}{$\begin{array}{l}\text { Nombre de } \\
\text { diferencial }\end{array}$} & \multicolumn{3}{|c|}{ Raza/aislado } & \multicolumn{4}{|c|}{ Aislados usados por varios autores } \\
\hline & \multirow{2}{*}{$\begin{array}{l}\text { Raza } 0 \text { Foc-71 } \\
\text { (Pénjamo) }\end{array}$} & \multirow{2}{*}{$\begin{array}{l}\text { Raza 1B/C Foc-27 } \\
\text { (Salvatierra) }\end{array}$} & \multirow{2}{*}{$\begin{array}{l}\text { Raza } 6 \text { Foc-12 } \\
\text { (V. de Santiago) }\end{array}$} & \multirow{2}{*}{$\begin{array}{c}\text { Raza } 5 \\
\text { Sharma et al., } 2005\end{array}$} & Raza 0 & Raza 1B/C & Raza 6 \\
\hline & & & & & \multicolumn{3}{|c|}{ Jiménez- Fernández 2011} \\
\hline JG 62 & $\mathrm{R}$ & $\mathrm{S}^{\dagger}$ & $\mathrm{S}^{\dagger \dagger}$ & $S$ & $\mathrm{R}$ & S & $S$ \\
\hline P 2245 & $S$ & $\mathrm{~S}^{\dagger}$ & $S^{\dagger \dagger}$ & S & S & S & S \\
\hline Sanford & I & I & I & S & NA & NA & NA \\
\hline CRIL 1-53 & $\mathrm{R}$ & I & I & $\mathrm{R}$ & NA & NA & NA \\
\hline CRIL 1-94 & S & S & S & I & NA & NA & NA \\
\hline CRIL 1-17 & $\mathrm{R}$ & $\mathrm{R}$ & $\mathrm{R}$ & $\mathrm{R}$ & NA & NA & NA \\
\hline CRIL 1-36 & $\mathrm{R}$ & $\mathrm{R}$ & I & $\mathrm{R}$ & NA & NA & NA \\
\hline WR 315 & $\mathrm{R}$ & $\mathrm{R}$ & $\mathrm{R}$ & $\mathrm{R}$ & $\mathrm{R}$ & $\mathrm{R}$ & $\mathrm{R}$ \\
\hline
\end{tabular}

$\mathrm{R}=$ resistente (0 a $10 \%) ; \mathrm{I}=$ intermedia (11 a $50 \%) ; \mathrm{S}=$ susceptible (50>); NA = datos no analizados; ${ }^{\dagger}$ Susceptible al amarillamiento; ${ }^{\dagger \dagger}$ Susceptible a marchitez.

Las razas predominantes en los cinco municipios muestreados fueron la 0 y la $1 \mathrm{~B} / \mathrm{C}$ que inducen el síndrome de amarillez vascular; las razas 4 y 6 que causan el síndrome de marchitez vascular, fueron identificadas en menor proporción. Los municipios Pénjamo, Salvatierra y Celaya mostraron mayor número de razas de FOC.

\section{AGRADECIMIENTOS}

Al Instituto Nacional de Investigaciones Forestales Agrícolas y Pecuarias por el apoyo económico para conducir la investigación: "Desarrollo de líneas resistentes al complejo de pudriciones radiculares en garbanzo, con énfasis en $F u$ sarium oxysporum f. sp. ciceris".

\section{BIBLIOGRAFÍA}

Arvayo-Ortiz R. M., M. Esqueda, E. Acedo-Félix, A. Sánchez and A. Gutiérrez (2011) Morphological variability and races of Fusarium oxysporum f. sp. ciceris associated with chickpea (Cicer arietinum) crops. American Journal Agricultural Biological Sciences 6:114-121.

Arvayo-Ortiz R. M., M. Esqueda, E. Acedo-Félix, H. González and G. Vargas (2012) New lines of chickpea againts Fusarium oxysporum f. sp. ciceris Wilt. American Journal of Applied Sciencies 9:686-693.

Don R. H., P. T. Cox, B. J. Wainwright, K. Baker and J. S. Mattick (1991) 'Touch-down' PCR to circumvent spurious priming during gene amplification. Nucleic Acids Research 19:4008.

Fehr W. R. (1987) Principles of Cultivar Development. Theory and Technique. Macmillan Publishing Company New York, USA. 536 p.

Jiménez-Díaz R. M., A. Trapero-Casas and J. Cabrera de la Colina (1989) Races of Fusarium oxysporum f. sp. ciceris infecting chickpea in southern Spain. In Vascular Wilt Diseases of Plants. E. C. Tjamos and C. H. Beckman (eds.). NATO ASI Series, Vol. H28. Springer-Verlag, Berlin. pp:515-520.

Jiménez-Gasco M. M., E. Pérez-Aretes and R. M. Jiménez-Díaz (2001) Identification of pathogenic races $0,1 \mathrm{~B} / \mathrm{C}, 5$, and 6 of Fusarium oxysporum f. sp. ciceris with random amplified polymorphic DNA (RAPD). European Journal of Plant Pathology 107:237248.

Jiménez-Gasco M. M. and R. M. Jiménez-Díaz (2003) The Fusarium oxysporum f. sp. ciceris/Cicer arietinum pathosystem: A case study of the evolution of plant-pathogenic fungi into races and pathotypes. International Microbiology 7:95-104.

Jiménez-Fernández D. M., Montes-Borrego, R. M. Jiménez-Díaz, J. A. Navas-Cortés and B. B. Landa (2011) In planta and soil quantification of Fusarium oxysporum f. sp. ciceris and evaluation of Fusarium wilt resistance in chickpea with a newly developed quantitative polymerase chain reaction assay. Phytopathology 101:250-262.

Haware M. P. and Y. L. Nene (1982) Races of Fusarium oxysporum f. sp. ciceris. Plant Disease 66:809-810.

Leslie J. F. and B. A. Summerell (2006) The Fusarium Laboratory Manual. First ed. Blackwell Publishing, Ames, IA, USA. 388 p.

Massa L. D. B. (1991) Preservacao de espécies de Fusarium sub óleo mineral. Pesquisa Agropecuaria Brasileira 26:853-855.

Nirenberg H. (1976) Untersuchungen uber die motphologische und biologische differenzierung in der Fusarium-Sektion Liseola. Mitteilungen aus der Biologischen Bundesanstait für Land-und Forstwirtschaft Berlin-Dahlem 169:1-117.

Phillips J. C. (1988) A distinct race of chickpea wilt in California. International Chickpea News 18:19-20.

Raeder U. and P. Broda (1985) Rapid DNA preparation from filamentous fungi. Letters of Applied Microbiology 1:17-20.

Schoonhoven V. A. y M. A. Pastor-Corrales (1987) Sistema Estándar para la Evaluación de Germoplasma de Frijol. Centro Internacional de Agricultura Tropical. Cali, Colombia 56 p.

Sharma K. D., P. Winter, G. Kahl and F. J. Muehlbauer (2004) Molecular mapping of Fusarium oxysporum f. sp. ciceris race 3 resistance gene in chickpea. Theoretical Applied Genetics 108:1243-1248.

Sharma K., D. Chen and F. J. Muehlbauer (2005) Genetics of chickpea resistance to five races of Fusarium wilt and a concise set of race differentials for Fusarium oxysporum f. sp. ciceris. Plant Disease 89:385-390.

Sharma K. D. and F. J. Muehlbauer (2007) Review Fusarium wilt of chickpea: physiological specialization, genetics of resistance and resistance gene tagging. Euphytica 157:1-14.

Sivaramakrishnan S., S. Kannan and S. D. Singh (2002) Genetic variability of Fusarium wilt pathogen isolates of chickpea (Cicer arietinum L.) assessed by molecular markers. Mycopathology 155:171-178.

Soltero D. L., A. E. Andrade, O. A. Grageda y V. J. Pérez (2008) San Antonio 05, variedad de garbanzo forrajero para la región Cienéga de Chapala México. Agricultura Técnica en México 34: 263-265.

Tekeoglu M, A. Tullu, W. J. Kaiser and F. J. Muehlbauer (2000) Inheritance and linkage of two genes that confer resistance to Fusarium wilt in Chickpea. Crop Science 40:1247-1251.

Trapero-Casas A. and R. M. Jiménez-Díaz (1985a) Fungal wilt and root trot diseases of chickpea in southern Spain. Phytopathology 75:1146-1151.

Trapero-Casas A. y R. M. Jiménez-Díaz (1985b) Etiología, importancia y 
distribución de la seca del garbanzo en el valle de Guadalquivir. Boletin de Sanidad Vegetal Plagas 11:69-145.

Tullu A., F. J. Muehlbauer, C. J. Simon, M. S. Mayer, J. Kumar, W. J. Kai- ser and J. M Kraft (1998) Inheritance and linkage of a gene for resistance to race 4 of Fusarium wilt and RAPD markers in chickpea. Euphytica 102:227-232. 